WINPEC Working Paper Series No.E1418

January2015

\title{
A new basis and the Shapley value
}

Koji Yokote, Yukihiko Funaki, Yoshio Kamijo

Waseda INstitute of Political EConomy

Waseda University

Tokyo,Japan 


\title{
A new basis and the Shapley value
}

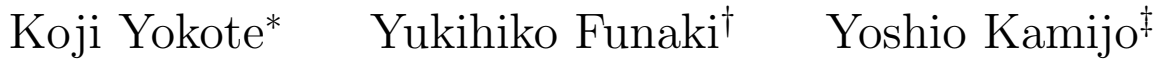

January 23, 2015

\begin{abstract}
The purpose of this paper is to introduce a new basis of the set of all TU games. Shapley (1953) introduced the unanimity game in which cooperation of all players in a given coalition yields payoff. We introduce the commander game in which only one player in a given coalition yields payoff. The set of the commander games forms a basis and has two properties. First, when we express a game by a linear combination of the basis, the coefficients related to singletons coincide with the Shapley value. Second, the basis induces the null space of the Shapley value.
\end{abstract}

JEL classification: C71

Keywords: TU game; Shapley value; Basis; Null space

\section{Introduction}

The basis consists of the unanimity games (Shapley (1953)) has long been recognized as a useful tool for the analysis of TU cooperative game theory. The basis is often used in the proof of axiomatization of the (weighted) Shapley value; see Young (1985), Chun (1989), Kalai and Samet (1987) or van den Brink (2002). The coefficient in the linear combination of the unanimity games is called the dividend. The class of games with nonnegative dividends was investigated by Llerena and Rafels (2006) or van den Brink et al. (2014).

*JSPS Research Fellow. Graduate School of Economics, Waseda University, 1-6-1, Nishi-Waseda, Shinjuku-ku, Tokyo 169-8050, Japan (sidehand@toki.waseda.jp)

${ }^{\dagger}$ Faculty of Political Science and Economics, Waseda University, 1-6-1, Nishi-Waseda, Shinjuku-ku, Tokyo 169-8050, Japan (funaki@waseda.jp)

${ }^{\ddagger}$ Kochi University of Technology, Department of Management, 185 Miyanokuchi, Tosayamada-Cho, Kami-Shi, Kochi 782-8502, Japan 
Since the set of TU games has a linear structure, to consider a basis is an essential task. The purpose of this paper is to introduce a new basis and explore its properties.

In the unanimity game, cooperation of all players in a given coalition yields payoff. In this paper, we introduce the commander game in which only one player in a given coalition yields payoff. The set of the commander games forms a basis and has two properties. First, when we express a game by a linear combination of the new basis, the coefficients related to singletons coincide with the Shapley value. Second, the basis induces the null space of the Shapley value: the set of games to which the Shapley value assigns 0 vector.

The payoff vector of each commander game can be uniquely determined by using three axioms: efficiency, equal treatment property and null player property. Thus, we can use the basis in the proof of axiomatization of the Shapley value. In addition, by using the two properties of the new basis, we can solve the following inverse problem: to characterize the class of games in which the Shapley value is equal to a fixed vector.

Some previous works on TU game discussed a way to express a game by using a specific class of games. Llerena and Rafels (2006) show that any TU game is expressed as the maximum of a finite collection of almost positive games. In the context of voting game, O'Neil and Peleg (2008) show five ways to combine two proper games into one game where the winning of a coalition depends on the two component games. The new basis in this paper also makes a contribution to this stream of research. We can express a game by a linear combination of games where the Shapley value is equal to 0 .

The new basis is also applicable to other research topics of the Shapley value. The basis enables us to give a new axiomatization of the weighted Shapley value; see Yokote (2014). Moreover, the basis can be used to investigate the relationship between the Shapley value and other solutions; see Yokote et al. (2013a).

This paper is organized as follows. Section 2 contains notations and definitions. In Section 3, we define the commander game and show that the set of the games is a basis. We also prove the first property of the new basis. In Section 4, we discuss the new basis from Shapley's (1953) axioms and prove the second property. In Section 5, we extend the new basis and deal with variants of the Shapley value. 


\section{Notations and definitions}

For two sets $A$ and $B, A \subseteq B$ means that $A$ is a subset of $B$. $A \subset B$ means that $A \subseteq B$ and $A \neq B$. Let $|A|$ denote the cardinality of $A . N \subset \mathbb{N}$ denotes a finite set of players, and we call $S \subseteq N$ a coalition of $N$. We define $|N|=n$. A characteristic function $v: 2^{N} \rightarrow \mathbb{R}$ assigns a real number to each coalition of $N$. We assume $v(\emptyset)=0$. We call $v(S)$ the worth of coalition $S$. A pair of $(N, v)$ is called a TU cooperative game, or simply a game. In the remaining part, we fix player set $N$ and write $v$ instead of $(N, v)$. Let $\Gamma^{N}$ denote the set of all games with player set $N$. We say that $v \in \Gamma^{N}$ is a simple game if $v(S)=0$ or 1 for all $S \subseteq N$. We regard $\Gamma^{N}$ as a linear space $\mathbb{R}^{2^{n}-1}$ by defining addition and scalar multiplication as follows: for any $v$, $w \in \Gamma^{N}$ and $\alpha \in \mathbb{R}$, we define $v+w$ and $\alpha v$ by $(v+w)(S)=v(S)+w(S)$ and $(\alpha v)(S)=\alpha v(S)$ for all $S \subseteq N$.

A solution function is a function from $\Gamma^{N}$ to $\mathbb{R}^{n}$. We define the Shapley value, introduced by Shapley (1953), as follows: for any $v \in \Gamma^{N}$,

$$
\phi_{i}(v)=\sum_{S \subseteq N: i \in S} \frac{(n-|S|) !(|S|-1) !}{n !}(v(S)-v(S \backslash\{i\})) \text { for all } i \in N .
$$

We can also calculate the Shapley value by using the dividend introduced by Harsanyi (1959). For any $v \in \Gamma^{N}$ and $S \subseteq N, S \neq \emptyset$, we define the dividend as follows:

$$
D(S, v)=\sum_{T \subseteq S}(-1)^{|S \backslash T|} v(T) .
$$

The following equation holds: for any $v \in \Gamma^{N}$,

$$
\phi_{i}(v)=\sum_{S \subseteq N: i \in S} \frac{1}{|S|} D(S, v) \text { for all } i \in N .
$$

Mathematically, the Shapley value $\phi$ is a surjective ${ }^{1}$ linear mapping from $\mathbb{R}^{2^{n}-1}$ to $\mathbb{R}^{n}$. The mapping $\phi$ has the null space defined by

$$
\left\{v \in \Gamma^{N}: \phi(v)=\mathbf{0}\right\} .
$$

The null space is the set of all games to which the Shapley value assigns 0 vector. The dimension of the space is equal to $2^{n}-1-n$.

\footnotetext{
${ }^{1}$ Surjective is due to the following inessential game property: let $x \in \mathbb{R}^{n}$ and consider the game $v$ such that $v(S)=\sum_{i \in S} x_{i}$ for all $S \subseteq N, S \neq \emptyset$. Then, $\phi(v)=x$.
} 


\section{New basis}

The purpose of this section is to define a new set of games and show that the set is a basis. We first revisit the set of the unanimity games introduced by Shapley (1953). Let $T \subseteq N, T \neq \emptyset$. We define the unanimity game $u_{T}$ as follows:

$$
u_{T}(S)=\left\{\begin{array}{l}
1 \text { if } T \subseteq S \\
0 \text { otherwise }
\end{array}\right.
$$

In the unanimity game, the cooperation of all players in $T$ yields payoff. When we express a game $v \in \Gamma^{N}$ by a linear combination of $\left\{u_{T}\right\}_{\emptyset \neq T \subseteq N}$, the coefficient of $u_{T}$ is equal to the dividend $D(T, v)$.

We now define a new game, which is the main research target of this paper. Let $T \subseteq N, T \neq \emptyset$. We define the game $\bar{u}_{T}$ as follows:

$$
\bar{u}_{T}(S)= \begin{cases}1 & \text { if }|S \cap T|=1 \\ 0 & \text { otherwise }\end{cases}
$$

From the definition, $\bar{u}_{T}$ is a simple game. We call $\bar{u}_{T}$ commander game. We consider the following situation behind the game. Each member in $T$ is a commander and has authority to control other players. If a coalition that includes only one member in $T$ forms, then the member behaves as a commander. The coalition obtains power, which results in the payoff of 1 . On the other hand, if a coalition that includes two or more members in $T$ forms, then they compete with each other and the coalition obtains nothing.

In order to prove that the set of the commander games is a basis, we prove a lemma.

Lemma 1 Let $T \subseteq N, T \neq \emptyset$. Then, we have

$$
|T| u_{T}=\sum_{S \subseteq T: S \neq \emptyset}(-1)^{|S|-1} \bar{u}_{S} .
$$

Proof. Let an arbitrary coalition $R \subseteq N$ be given. We calculate the worth of coalition $R$ in the right-hand side. From the definition of the commander game, we only need to consider a coalition $S \subseteq T$ such that $|S \cap R|=1$. Such a coalition $S$ can be determined by choosing 1 player from $T \cap R$ and $k$ players from $T \backslash R$, where $0 \leq k \leq|T \backslash R|$. As a result, we obtain

$$
\sum_{S \subseteq T: S \neq \emptyset}(-1)^{|S|-1} \bar{u}_{S}(R)=|T \cap R| \cdot \sum_{k=0}^{|T \backslash R|}\left(\begin{array}{c}
|T \backslash R| \\
k
\end{array}\right)(-1)^{k}= \begin{cases}|T| & \text { if } T \subseteq R \\
0 & \text { otherwise }\end{cases}
$$


where the second equality holds from the binomial theorem. It follows that the worth of coalition $R$ in the right-hand side is equal to $|T| u_{T}(R)$.

Theorem 1 The set of games $\left\{\bar{u}_{T}\right\}_{\emptyset \neq T \subseteq N}$ is a basis of $\Gamma^{N}$.

Proof. Let $v \in \Gamma^{N}$. From the fact that the dividend is the coefficient in the linear combination of the unanimity games, we have

$$
\begin{aligned}
v & =\sum_{R \subseteq N: R \neq \emptyset} \frac{D(R, v)}{|R|} \cdot|R| u_{R} \\
& =\sum_{R \subseteq N: R \neq \emptyset} \frac{D(R, v)}{|R|} \cdot \sum_{T \subseteq R}(-1)^{|T|-1} \bar{u}_{T} \\
& =\sum_{T \subseteq N: T \neq \emptyset}(-1)^{|T|-1} \sum_{R \subseteq N: T \subseteq R} \frac{D(R, v)}{|R|} \bar{u}_{T},
\end{aligned}
$$

where the second equality holds from Lemma 1 . As a result, any game $v \in \Gamma^{N}$ can be expressed by a linear combination of the games $\left\{\bar{u}_{T}\right\}_{\emptyset \neq T \subseteq N}$. In other words, the set $\left\{\bar{u}_{T}\right\}_{\emptyset \neq T \subseteq N}$ spans $\Gamma^{N}$. If the set $\left\{\bar{u}_{T}\right\}_{\emptyset \neq T \subseteq N}$ is linearly dependent, then there exist a coalition $T \subseteq N, T \neq \emptyset$, and a vector $\left(\alpha_{S}\right)_{\emptyset \neq S \subseteq N, S \neq T}$ such that

$$
\bar{u}_{T}=\sum_{S \subseteq N: S \neq \emptyset, S \neq T} \alpha_{S} \bar{u}_{S} .
$$

Together with equation (1), the set $\Gamma^{N}$ can be spanned by vectors with less than $2^{n}-1$ vectors, which is a contradiction. ${ }^{2}$

For any $v \in \Gamma^{N}$, let $d(T, v)$ denote the coefficient in the linear combination of $\left\{\bar{u}_{T}\right\}_{\emptyset \neq T \subseteq N}$, namely, $v=\sum_{T \subseteq N: T \neq \emptyset} d(T, v) \bar{u}_{T}$. From equation (1), we obtain the following proposition:

Proposition 1 For any $v \in \Gamma^{N}$,

$$
d(\{i\}, v)=\sum_{R \subseteq N: i \in R} \frac{D(R, v)}{|R|}=\phi_{i}(v) \text { for all } i \in N .
$$

Proposition 1 states that the coefficients related to singletons coincide with the Shapley value. We will revisit this property in the next section.

\footnotetext{
${ }^{2}$ Recall the following result in linear algebra: if the vectors $x_{1}, \cdots, x_{n}$ span a linear space $X$ and the vectors $y_{1}, \cdots, y_{j}$ in $X$ are linearly independent, then $j \leq n$. See Lax (2007), Lemma 1 on page 5 .
} 
Remark 1 Equation (1) explains how to calculate the coefficient $d(T, v)$ by using the dividend. We can also calculate the coefficient by using the potential function $P(T, v)$, introduced by Hart and Mas-Colell (1989), as follows:

$$
d(T, v)=\sum_{S: N \backslash T \subseteq S}(-1)^{|S \cap T|-1} P(S, v) \text { for all } T \subseteq N, T \neq \emptyset .
$$

For the proof, see Yokote et al. (2013b). From the above equation, $d(T, v)$ is expressed by $P(T, v)$. In addition, $P(T, v)$ can be expressed by the dividend $D(T, v) .{ }^{3}$ These results explain the relationship between the three real-valued functions, $d(T, v), P(T, v)$ and $D(T, v)$.

\section{Basis and Shapley's axioms}

The unanimity game is useful in that the payoff vector of the game can be uniquely determined by using axioms. In this section, we show that the commander game has the same property. We introduce additional notations. Let $v \in \Gamma^{N}$ and $i, j \in N, i \neq j$, be given. We say that $i$ and $j$ are substitutes in $v$ if $v(S \cup\{i\})-v(S)=v(S \cup\{j\})-v(S)$ for all $S \subseteq N \backslash\{i, j\}$. We say that $i$ is a null player in $v$ if $v(S \cup\{i\})-v(S)=0$ for all $S \subseteq N \backslash\{i\}$.

Shapley (1953) characterized the Shapley value by using the following axioms satisfied by a solution function $\psi$ :

Efficiency $\sum_{i \in N} \psi_{i}(v)=v(N)$ for all $v \in \Gamma^{N}$.

Equal Treatment Property If $i$ and $j$ are substitutes in $v \in \Gamma^{N}$, then $\psi_{i}(v)=\psi_{j}(v)$.

Null Player Property If $i$ is a null player in $v \in \Gamma^{N}$, then $\psi_{i}(v)=0$.

Additivity For any $v, w \in \Gamma^{N}$, we have $\psi(v+w)=\psi(v)+\psi(w)$.

By using the first three axioms, we can calculate the Shapley value in the game $\bar{u}_{T}, T \subseteq N,|T| \geq 2$. First, consider a player $j \in N \backslash T$. Then, for any coalition $S \subseteq N \backslash\{j\}$, we have $|S \cap T|=|(S \cup\{j\}) \cap T|$. Thus, $j$ is a null player, which implies $\phi_{j}\left(\bar{u}_{T}\right)=0$ from Null Player Property. From Efficiency, $\sum_{i \in T} \phi_{i}\left(\bar{u}_{T}\right)=0$. Since any two players in $T$ are substitutes, from Equal Treatment Property, we have $\phi_{i}\left(\bar{u}_{T}\right)=0$ for all $i \in T$. It follows that $\phi\left(\bar{u}_{T}\right)=\mathbf{0}$. Note that we can also determine the payoff vector in the game $\bar{u}_{\{i\}}, i \in N$, in the same way.

\footnotetext{
${ }^{3}$ See equation (2.3) of Hart and Mas-Colell (1989).
} 
Since the set $\left\{\bar{u}_{T}: T \subseteq N,|T| \geq 2\right\}$ consists of $2^{n}-1-n$ linearly independent vectors, we obtain the following theorem:

Proposition 2 The set $\left\{\bar{u}_{T}: T \subseteq N,|T| \geq 2\right\}$ spans the null space of $\phi$.

Proposition 2 states that the Shapley value does not depend on the coefficient $d(T, v), T \subseteq N,|T| \geq 2$. Recall that from Proposition 1, the coefficients $d(\{i\}, v), i \in N$, coincide with the Shapley value. As a consequence, we obtain the following corollary:

Corollary 1 Let $x \in \mathbf{R}^{n}$. Then, $\phi(v)=x$ if and only if there exists a vector $\left(\alpha_{T}\right)_{T \subseteq N:|T| \geq 2} \in \mathbb{R}^{2^{n}-1-n}$ such that

$$
v=\sum_{i \in N} x_{i} \bar{u}_{\{i\}}+\sum_{T \subseteq N:|T| \geq 2} \alpha_{T} \bar{u}_{T}
$$

In Corollary 1, we characterize the set of all games to which the Shapley value assigns a fixed vector. This approach is known as the inverse problem. By solving the problem, we can characterize the equivalence relation that relates two different games with the same Shapley value. If two games are equivalent by the relation, we can know that the Shapley value is silent about the difference between the two situations described by the games.

Example 1 By using Corollary 1, we obtain the following result for 3-person games: let $v \in \Gamma^{N}, N=\{1,2,3\}$. Then, $\phi(v)=x$ if and only if there exists $\left(y_{12}, y_{13}, y_{23}, y_{N}\right) \in \mathbb{R}^{4}$ such that $v(N)=x_{1}+x_{2}+x_{3}$ and

$$
v(\{i, j\})=x_{i}+x_{j}+y_{i k}+y_{j k}, v(\{k\})=x_{k}+y_{i k}+y_{j k}+y_{N},
$$

where $i, j, k$ are distinct players in $N$. The above equations imply

$$
v(\{i, j\})=x_{i}+x_{j}+v(\{k\})-x_{k}-y_{N} .
$$

As a result, we obtain the following: let $v \in \Gamma^{N}, N=\{1,2,3\}$, be a game such that $v(\{k\})=0$ for all $k \in N$. Then, $\phi(v)=x$ if and only if there exists $y \in \mathbb{R}$ such that $v(N)=x_{1}+x_{2}+x_{3}$ and

$$
v(\{i, j\})=x_{i}+x_{j}-x_{k}+y,
$$

where $i, j, k$ are distinct players in $N$. The only if part says that, given an arbitrary vector $x$, we can always find an identical amount $y$ for all coalitions with 2 players. 
The null space or the inverse problem have been investigated in previous works. As for the null space, Kleinberg and Weiss (1985) give a direct sum decomposition of the space. Dragan et al. (1989) characterized the space by using the basis related to the potential function by Hart and Mas-Colell (1989). As for the inverse problem, see Dragan (2005) or Dragan (2012). The merit of using the new basis is that we can solve the above problems by using only simple games.

\section{Basis of variants of the Shapley value}

By slightly changing the definition of the new basis, we can deal with variants of the Shapley value. We first consider the weighted Shapley value. For any $w \in \mathbb{R}_{++}^{n}$, we define the weighted Shapley value with positive weight $w$ as follows: for any $v \in \Gamma^{N}$,

$$
\phi_{i}^{w}(v)=\sum_{S \subseteq N: i \in S} D(S, v) \cdot \frac{w_{i}}{\sum_{j \in S} w_{j}} \text { for all } i \in N .
$$

For any $w \in \mathbb{R}_{++}^{n}$ and $T \subseteq N, T \neq \emptyset$, we define $\bar{u}_{T}^{w} \in \Gamma^{N}$ as follows:

$$
\bar{u}_{T}^{w}(S)= \begin{cases}w_{i}, i \in T \cap S & \text { if }|S \cap T|=1, \\ 0 & \text { otherwise. }\end{cases}
$$

Remark 2 (Theorem 2 of Yokote (2014)) Let $w \in \mathbb{R}_{++}^{n}$. Then, the set of games $\left\{\bar{u}_{\{i\}}: i \in N\right\} \cup\left\{\bar{u}_{T}^{w}:|T| \geq 2\right\}$ is a basis of $\Gamma^{N}$. When we express a game $v \in \Gamma^{N}$ by a linear combination of this basis, the coefficient of $\bar{u}_{\{i\}}$ is equal to $\phi_{i}^{w}(v)$ for all $i \in N$. Moreover, the set of games $\left\{\bar{u}_{T}^{w}: T \subseteq N,|T| \geq\right.$ $2\}$ spans the null space of $\phi^{w}$.

Next, we consider another variant. Let a player set $N=\{1, \cdots, n\}$ be given. Consider a function $f:\{1, \cdots, n\} \rightarrow \mathbb{R} \backslash\{0\}$. We define $v^{f} \in \Gamma^{N}$ as follows:

$$
v^{f}(S)=f(|S|) v(S) \text { for all } S \subseteq N, S \neq \emptyset .
$$

We define a new solution function $\phi^{f}$ by $\phi^{f}(v)=\phi\left(v^{f}\right)$ for all $v \in \Gamma^{N}$. For any $T \subseteq N, T \neq \emptyset$, we define $\bar{u}_{T}^{f}$ as follows:

$$
\bar{u}_{T}^{f}(S)= \begin{cases}1 / f(|S|) & \text { if }|S \cap T|=1, \\ 0 & \text { otherwise. }\end{cases}
$$


Remark 3 Let $f:\{1, \cdots, n\} \rightarrow \mathbb{R} \backslash\{0\}$. Then, the set of games $\left\{\bar{u}_{T}^{f}: T \subseteq\right.$ $N, T \neq \emptyset\}$ is a basis of $\Gamma^{N}$. When we express a game $v \in \Gamma^{N}$ by a linear combination of this basis, the coefficient of $\bar{u}_{\{i\}}^{f}$ is equal to $\phi_{i}^{f}(v)$ for all $i \in N$. Moreover, the set of games $\left\{\bar{u}_{T}^{f}:|T| \geq 2\right\}$ spans the null space of $\phi^{f}$.

We skip the proof since it is straightforward from $\left(\bar{u}_{T}^{f}\right)^{f}=\bar{u}_{T}$ for all $T \subseteq N$, $T \neq \emptyset$. For any $\delta \in(0,1]$, by letting $f(x)=\delta^{n-x}$, $\phi^{f}$ is the discounted Shapley value with discount factor $\delta$ introduced by Joosten (1996). We can also determine the payoff vector in the game $\bar{u}_{T}^{f}$ by using efficiency, equal treatment property and $\delta$-reducing player property introduced by van den Brink and Funaki (2010).

\section{References}

[1] Chun, Y. (1989): "A new axiomatization of the Shapley value," Games and Economic Behavior, 1(2), 119-130.

[2] Dragan, I. (2005): "On the inverse problem for semivalues of cooperative TU games," International Journal of Pure and Applies Mathematics 22: 539-555.

[3] Dragan I. (2012): "On the inverse problem for multiweighted Shapley values of cooperative TU games. Int J Pure and Appl Math 75: 279-287

[4] Dragan, I., J. Potters and S. Tijs (1989): "Superadditivity for solutions of coalitional games," Libertas Mathematica 9: 101-110.

[5] Harsanyi, J. C. (1959): "A bargaining model for cooperative n-person games," In: Tucker AW, Luce RD (ed) Contributions to the theory of games IV. Princeton University Press, Princeton, pp 325-355.

[6] Hart, S., and A. Mas-Colell (1989): "Potential, value and consistency," Econometrica 57: 589-614.

[7] Joosten, R. (1996): "Dynamics, equilibria and values," Dissertation, Maastricht University.

[8] Kalai, E., and D. Samet (1987): "On weighted Shapley values," International Journal of Game Theory, 16(3), 205-222.

[9] Kleinberg, N. L., and J. H. Weiss (1985): "Equivalent N-person games and the null space of the Shapley value," Mathematics of Operations Research 10: 233-243. 
[10] Lax, P. D. (2007) Linear algebra and its applications, 2nd edn. Wiley, New Jersey

[11] Llerena, F., and C. Rafels (2006): "The vector lattice structure of the n-person TU games," Games and Economic Behavior, 54(2), 373-379.

[12] O'Neill, B., and B. Peleg (2008): "Lexicographic composition of simple games," Games and Economic Behavior, 62(2), 628-642.

[13] Shapley, L. S. (1953): "A value for n-person games," In: Roth AE (ed) The Shapley value. Cambridge University Press, Cambridge, pp 41-48.

[14] van den Brink, R. (2002): "An axiomatization of the Shapley value using a fairness property," International Journal of Game Theory, 30(3), 309-319.

[15] van den Brink, R., and Y. Funaki (2010): "Axiomatization and implementation of discounted Shapley values," Tinbergen Institute Discussion Paper 2010-065/1, Tinbergen Institute and VU University, Amsterdam.

[16] van den Brink, R., G. van der Laan and V. A. Vasil'ev (2014): "Constrained core solutions for totally positive games with ordered players," International Journal of Game Theory, 43(2), 351-368.

[17] Yokote, K. (2014): "Weak Addition Invariance and axiomatization of the weighted Shapley value," International Journal of Game Theory. doi:10.1007/s0018201404297

[18] Yokote, K., Y. Funaki, and Y. Kamijo (2013a): "Relationship between the Shapley value and other solution concepts," Institute of Research in Contemporary Political and Economic Affairs Working Paper No. E1304, Waseda University. http://www.wasedapse.jp/ircpea/en/publications/working-paper-e-series/ Accessed 17 January 2015

[19] Yokote, K., Y. Funaki, and Y. Kamijo (2013b): "Linear basis approach to the Shapley value," Institute of Research in Contemporary Political and Economic Affairs Working Paper No.E1303, Waseda University. http://www.waseda-pse.jp/ircpea/en/publications/working-paper-eseries/ Accessed 17 January 2015

[20] Young, H. P. (1985): "Monotonic solutions of cooperative games," International Journal of Game Theory, 14(2), 65-72. 